\title{
FUZZY FILTERS IN BCI-ALGEBRAS
}

\author{
C. LELE, C. WU, and T. MAMADOU
}

Received 9 December 2000

\begin{abstract}
We introduce the notion of fuzzy filters and weak filters in BCI-algebras and discuss their properties. Then we establish some relations among filters, fuzzy filters, and weak filters in BCI-algebras.
\end{abstract}

2000 Mathematics Subject Classification: 06F35.

1. Introduction. The main problem in fuzzy mathematics is how to carry out the ordinary concepts to the fuzzy case. The difficulty lies in how to pick out the rational generalization from the large number of available approaches. It is worth noting that fuzzy ideals (fuzzy filters) are different from ordinary ideals (filters) in the sense that one cannot say which BCI-algebra element belongs to the fuzzy ideal (fuzzy filter) under consideration and which one does not.

In $[9,8,10,11,12]$, some properties of fuzzy semigroups and ideals in BCI-algebras were established using the concept of fuzzy point. In this note, we introduce and give some characterization of fuzzy filter in BCI-algebras.

1.1. Preliminaries. (See $[1,4,5,6,13]$.) An algebra $(X, *, 0)$ of type $(2,0)$ is said to be a BCI-algebra if for any $x, y, z$ in $X$, the following conditions hold:

(BCI-1) $((x * y) *(x * z)) *(z * y)=0$.

(BCI-2) $(x *(x * y)) * y=0$.

(BCI-3) $x * x=0$.

(BCI-4) $x * y=0$ and $y * x=0$ imply $x=y$.

If we define a binary relation $\leq$ on $X$ by

(BCI-5) $x \leq y$ if and only if $x * y=0$, then $(X, \leq)$ is partially ordered.

The following properties also hold in any BCI-algebra (see [1, 2, 3, 4, 5, 6, 7, 13]).

(1) $x * 0=x$.

(2) $x * y=0$ implies $(x * z) *(y * z)=0$ and $(z * y) *(z * x)=0$.

(3) $(x * y) * z=(x * z) * y$.

(4) $(x * y) * x=0$.

(5) $x *(x *(x * y))=x * y$.

(6) $0 *(x * y)=(0 * x) *(0 * y)$.

(7) $(x \Lambda y) * y=0$, where $x \Lambda y=x *(x * y)$.

1.2. Algebra structure of the set of fuzzy points in BCI-algebras. Let $(X, *, 0)$ be a BCI-algebra. A fuzzy set $A$ in $X$ is a map $A: X \rightarrow[0,1]$. If $\xi$ is the family of all fuzzy sets in $X, x_{\lambda} \in \xi$ is a fuzzy point if and only if $x_{\lambda}(y)=\lambda$ when $x=y$; and $x_{\lambda}(y)=0$ when $x \neq y$. We denote by $\tilde{X}=\left\{x_{\lambda} / x \in X, \lambda \in(0,1]\right\}$ the set of all fuzzy points on $X$ 
and define a binary operation on $\tilde{X}$ as follows:

$$
x_{\lambda} * y_{\mu}=(x * y)_{\min (\lambda, \mu)}
$$

It is easy to verify that $(\tilde{X}, *)$ satisfies the following conditions: for any $x_{\lambda}, y_{\mu}, z_{\alpha} \in \tilde{X}$

$\left(\mathrm{BCI}-1^{\prime}\right)\left(\left(x_{\lambda} * y_{\mu}\right) *\left(x_{\lambda} * z_{\alpha}\right)\right) *\left(z_{\alpha} * y_{\mu}\right)=0_{\min (\lambda, \mu, \alpha)}$.

$\left(\mathrm{BCI}-2^{\prime}\right)\left(x_{\lambda} *\left(x_{\lambda} * y_{\mu}\right)\right) * y_{\mu}=0_{\min (\lambda, \mu)}$.

(BCI-3') $x_{\lambda} * x_{\lambda}=0_{\lambda}$.

REMARK 1.1. The condition (BCI-4) is not true in $(\tilde{X}, *)$. So the partial order $\leq$ in $X$ cannot be extended in $(\tilde{X}, *)$. We will call $(\tilde{X}, *)$ a weak BCI-algebra. We can also establish the following conditions: for any $x_{\lambda}, y_{\mu}, z_{\alpha} \in \tilde{X}$

(1') $x_{\lambda} * 0_{\mu}=x_{\min (\lambda, \mu)}$.

(2') $x_{\lambda} * y_{\mu}=0_{\min (\lambda, \mu)}$ implies that

$$
\left(x_{\lambda} * z_{\alpha}\right) *\left(y_{\mu} * z_{\alpha}\right)=0_{\min (\lambda, \mu, \alpha),} \quad\left(z_{\alpha} * y_{\mu}\right) *\left(z_{\alpha} * x_{\lambda}\right)=0_{\min (\lambda, \mu, \alpha)} .
$$

(3') $\left(x_{\lambda} * y_{\mu}\right) * z_{\alpha}=\left(x_{\lambda} * z_{\alpha}\right) * y_{\mu}$.

$\left(4^{\prime}\right)\left(x_{\lambda} * y_{\mu}\right) * x_{\lambda}=0_{\min (\lambda, \mu)}$.

(5') $x_{\lambda} *\left(x_{\lambda} *\left(x_{\lambda} * y_{\mu}\right)\right)=x_{\lambda} * y_{\mu}$.

(6') $0_{\alpha} *\left(x_{\lambda} * y_{\mu}\right)=\left(0_{\alpha} * x_{\lambda}\right) *\left(0_{\alpha} * y_{\mu}\right)$.

(7') $\left(x_{\lambda} \Lambda y_{\mu}\right) * y_{\mu}=0_{\min (\lambda, \mu)}$, where $x_{\lambda} \Lambda y_{\mu}=x_{\lambda} *\left(x_{\lambda} * y \mu\right)$.

We also recall that if $A$ is a fuzzy subset of a BCI-algebra $X$, then we have the following:

$$
\tilde{A}=\left\{x_{\lambda}: A(x) \geq \lambda, x_{\lambda} \in \tilde{X}, \lambda \in(0,1]\right\},
$$

for any $\lambda \in(0,1]$

$$
\tilde{X}_{\lambda}=\left\{x_{\lambda}: x_{\lambda} \in \tilde{X}\right\}, \quad \tilde{A}_{\lambda}=\left\{x_{\lambda}: x_{\lambda} \in \tilde{A}\right\} .
$$

We have $\tilde{X}_{\lambda} \subseteq \tilde{X}, \tilde{A} \subseteq \tilde{X}, \tilde{A}_{\lambda} \subseteq \tilde{A}$, and $\tilde{A}_{\lambda} \subseteq \tilde{X}_{\lambda}$.

It can easily be proved that $\left(\tilde{X}_{\lambda}, *, 0_{\lambda}\right)$ is a BCI-algebra.

\subsection{Weak ideal}

DefinItion 1.2 (see [7]). A nonempty subset $I$ of BCI-algebras $X$ is called an ideal if it satisfies

(a) $0 \in I$,

(b) $x * y \in I$ and $y \in I$ imply $x \in I$.

An ideal $I$ of $X$ is said to be closed if $0 * x \in I$ when $x \in I$.

Definition 1.3 (see [7]). A fuzzy subset $A$ of a BCI-algebra $X$ is a fuzzy ideal if and only if:

(a) For any $x \in X, A(0) \geq A(x)$.

(b) For any $x, y \in X, A(x) \geq \min (A(x * y), A(y))$.

A fuzzy ideal $A$ of $X$ is said to be closed if $A(0 * x) \geq A(x)$ for any $x \in X$. 
DEFINITION 1.4. $\tilde{A}$ is a weak ideal of $\tilde{X}$ if it satisfies the following conditions:

(a) For any $v \in \operatorname{Im}(A), 0_{v} \in \tilde{A}$.

(b) For any $x_{\lambda}, y_{\mu} \in \tilde{X}$ such that $x_{\lambda} * y_{\mu} \in \tilde{A}$ and $y_{\mu} \in \tilde{A}$, we have $x_{\min (\lambda, \mu)} \in \tilde{A}$. A weak ideal $\tilde{A}$ is said to be closed if $0_{\lambda} * x_{\lambda} \in \tilde{A}$ for any $x_{\lambda} \in \tilde{A}$.

REMARK 1.5. A weak ideal $\tilde{A}$ has the following property:

$$
x_{\lambda} * y_{\mu}=0_{\min (\lambda, \mu)}, \quad y_{\mu} \in \tilde{A} \Longrightarrow x_{\min (\lambda, \mu)} \in \tilde{A} .
$$

Clearly, let $x_{\lambda}, y_{\mu} \in \tilde{X}$ such that $x_{\lambda} * y_{\mu}=0_{\min (\lambda, \mu)}$ and $y_{\mu} \in \tilde{A}$.

Now, $y_{\mu} \in \tilde{A}$ implies that $A(y) \geq \mu$. Let $A(y)=\alpha$. Using Definition 1.4(a), we obtain $0_{\alpha} \in \tilde{A}$. So $A(0) \geq \alpha$. But $\alpha=A(y) \geq \mu \geq \min (\lambda, \mu)$. So $0_{\min (\lambda, \mu)} \in \tilde{A}$. Using Definition 1.4(b), we obtain $x_{\min (\lambda, \mu)} \in \tilde{A}$.

Now we discuss the relation between ideal, fuzzy ideal, and weak ideal.

THEOREM 1.6. Suppose that $A$ is a fuzzy subset of $X$. Then the following conditions are equivalent:

(1) $A$ is a closed fuzzy ideal.

(2) $\tilde{A}$ is a closed weak ideal.

(3) For any $t \in(0,1]$ the $t$-level subset $A^{t}=\{x \in X / A(x) \geq t\}$ is a closed ideal when $A^{t} \neq \varnothing$.

The proof is the same as [10, Theorem 1.4].

2. Fuzzy filter. In this section, we introduce and discuss the notion of fuzzy filter in a BCI-algebra $X$.

DeFinition 2.1 (see [1]). A filter of $X$ is a nonempty subset $F$ such that

(a) $x \in F$ and $y \in F$ imply $x \Lambda y \in F$ and $x \Lambda y \in F$.

(b) $x \in F$ and $x \leq y$ imply $y \in F$.

Definition 2.2. A nonconstant fuzzy set $A$ of $X$ is a fuzzy filter if:

(a) $A(x \Lambda y) \geq \min (A(x), A(y))$ and $A(y \Lambda x) \geq \min (A(x), A(y))$.

(b) $A(y) \geq A(x)$ when $x \leq y$.

DEFINITION 2.3. Let $F$ be a subset of $X$ and $\lambda \in(0,1]$ we define a fuzzy set $\lambda_{X_{F}}$ as $\lambda_{X F}(x)=\lambda$ if $x \in F$ and $\lambda \chi_{F}(x)=0$ otherwise.

THEOREM 2.4. A subset $F$ of $X$ is a filter if and only if $\lambda_{X_{F}}$ is a fuzzy filter.

Proof. Suppose that $F$ is a filter of $X$.

(a) Let $x, y \in X$ such that $x \in F$ and $y \in F$. We have $\lambda \chi_{F}(x)=\lambda$ and $\lambda \chi_{F}(y)=\lambda$. Since $F$ is a filter, we have then $x \Lambda y \in F$ and $y \Lambda x \in F$ and $\lambda \chi_{F}(x \Lambda y)=\lambda=\lambda \chi_{F}(y \Lambda x)$ such that

$$
\lambda \chi_{F}(x \Lambda y) \geq \min \left(\lambda \chi_{F}(x), \lambda x_{F}(y)\right), \quad \lambda x_{F}(y \Lambda x) \geq \min \left(\lambda \chi_{F}(x), \lambda \chi_{F}(y)\right) .
$$

If $x \notin F$ or $y \notin F, \min \left(\lambda_{\chi_{F}}(x), \lambda \chi_{F}(y)\right)=0$ such that

$$
\lambda \chi_{F}(x \Lambda y) \geq \min \left(\lambda \chi_{F}(x), \lambda \chi_{F}(y)\right), \quad \lambda \chi_{F}(y \Lambda x) \geq \min \left(\lambda \chi_{F}(x), \lambda \chi_{F}(y)\right) .
$$


(b) Let $x, y \in X$ and $x \leq y$, we must show that $\lambda \chi_{F}(y) \geq \lambda \chi_{F}(x)$.

If $x \in F$, we have $\lambda_{X_{F}}(x)=\lambda$, since $F$ is a filter $y \in F$ and $\lambda_{X_{F}}(y)=\lambda$ such that $\lambda \chi_{F}(y) \geq \lambda \chi_{F}(x)$. If $x \in F, \lambda \chi_{F}(x)=0$ and we obtain $\lambda \chi_{F}(y) \geq \lambda \chi_{F}(x)$.

Conversely, assume that $\lambda \chi_{F}$ is a fuzzy filter, we must prove that $F$ is a filter.

(a) Let $x, y \in F$. Then $\lambda_{X_{F}}(x)=\lambda_{X_{F}}(y)=\lambda$. Since $\lambda_{X_{F}}$ is a fuzzy filter,

$$
\begin{aligned}
& \lambda X_{F}(x \Lambda y) \geq \min \left(\lambda \chi_{F}(x), \lambda X_{F}(y)\right)=\lambda, \\
& \lambda X_{F}(y \Lambda x) \geq \min \left(\lambda X_{F}(x), \lambda X_{F}(y)\right)=\lambda .
\end{aligned}
$$

So $\lambda \chi_{F}(x \Lambda y)=\lambda \chi_{F}(y \Lambda x)=\lambda$ such that $x \Lambda y \in F$ and $y \Lambda x \in F$.

(b) Let $x \in F$ and $x \leq y$. Then $\lambda_{\chi_{F}}(x)=\lambda$ and because $\lambda_{X_{F}}$ is a fuzzy filter $\lambda_{X_{F}}(y) \geq$ $\lambda_{X_{F}}(x)$. So $\lambda_{\chi_{F}}(y)=\lambda$ such that $y \in F$.

We characterize fuzzy filters in terms of level subsets as in the following theorem.

THEOREM 2.5. A fuzzy subset $A$ of a BCI-algebra $X$ is a fuzzy filter if and only if for any $t \in(0,1]$ the $t$-level subset $A^{t}=\{x \in X / A(x) \geq t\}$ is a filter when $A^{t} \neq \varnothing$.

Proof. Suppose that for any $t \in(0,1]$ the $t$-level subset $A^{t}=\{x \in X / A(x) \geq t\}$ is a filter when $A^{t} \neq \varnothing$, we want to show that $A$ is a fuzzy filter.

(a) Let $x, y \in X$ and $t=\min (A(x), A(y))$. Then $x, y \in A^{t}$ and because $A^{t}$ is a filter, we have $x \Lambda y \in A^{t}$ and $y \Lambda x \in A^{t}$ such that $A(x \Lambda y) \geq t=\min (A(x), A(y))$ and $A(y \Lambda x) \geq t=\min (A(x), A(y))$.

(b) Let $x, y \in X$ and $x \leq y$, we must show that $A(y) \geq A(x)$.

Let $t=A(x)$. Then $x \in A^{t}$ and because $A^{t}$ is a filter, we have $y \in A^{t}$ such that $A(y) \geq t=A(x)$.

Conversely, assume that $A$ is a fuzzy filter of $X$, we must prove that for any $t \in(0,1]$ the $t$-level subset $A^{t}=\{x \in X / A(x) \geq t\}$ is a filter when $A^{t} \neq \varnothing$.

(a) Let $x, y \in A^{t}$. Then $A(x) \geq t$ and $A(y) \geq t$. Since $A$ is a fuzzy filter, $A(x \Lambda y) \geq$ $\min (A(x), A(y)) \geq t$ and $A(y \Lambda x) \geq \min (A(x), A(y)) \geq t$ such that $x \Lambda y \in A^{t}$ and $y \Lambda x \in A^{t}$.

(b) Let $x \in A^{t}$ and $x \leq y$. Then $A(x) \geq t$ and because $A$ is a fuzzy filter $A(y) \geq A(x)$. So $A(y) \geq A(x) \geq t$ such that $y \in A^{t}$.

Now, we construct new fuzzy filters from old ones.

Definition 2.6 (see [8]). If $A$ is a fuzzy subset of $X$ and $a \in[0,1)$, let $A^{a}: X \rightarrow[0,1]$ be given by $A^{a}(x)=(A(x))^{a}$.

THEOREM 2.7. If $A$ is a fuzzy filter, then $A^{a}$ is also a fuzzy filter.

Proof. (a) Let $x, y \in X$. $A^{a}(x \Lambda y)=(A(x \Lambda y))^{a}$. Since $A$ is a fuzzy filter, $A(x \Lambda y) \geq$ $\min (A(x), A(y))$ such that

$$
\begin{aligned}
(A(x \Lambda y))^{a} & \geq(\min (A(x), A(y)))^{a} \\
& =\min \left((A(x))^{a},(A(y))^{a}\right) \\
& =\min \left(A^{a}(x), A^{a}(y)\right) .
\end{aligned}
$$


So $A^{a}(x \Lambda y) \geq \min \left(A^{a}(x), A^{a}(y)\right)$. Similarly, we can prove that

$$
A^{a}(y \Lambda x) \geq \min \left(A^{a}(x), A^{a}(y)\right) .
$$

(b) Let $x, y \in X$ such that $x \leq y \cdot A^{a}(y)=(A(y))^{a}$. Since $A$ is a fuzzy filter, $A(y) \geq$ $A(x)$ such that

$$
(A(y))^{a} \geq(A(y))^{a}=A^{a}(x) .
$$

So $A^{a}(y) \geq A^{a}(x)$.

Definition 2.8 (see [7]). Let $f: X \rightarrow Y$ be a mapping. Let $B$ be a fuzzy subset in $f(X)$. Then $f^{-1}(B)(x)=B(f(x))$ is a fuzzy subset, conversely, let $A$ be a fuzzy subset in $X$, then $f(A)$, defined by

$$
f(A)(y)=\sup _{t \in f^{-1}(y)} A(t)
$$

is a fuzzy subset of $Y$.

A mapping $f$ is called a BCI-homomorphism if $f(x * y)=f(x) * f(y)$. It is easy to see that in a BCI-homomorphism, $f(0)=0$ and $f(x) \leq f(y)$ when $x \leq y$.

THEOREM 2.9. Let $f$ be an onto BCI-homomorphism.

(1) If $B$ is a fuzzy filter, then $f^{-1}(B)$ is also a fuzzy filter.

(2) If $A$ is a fuzzy filter and has a sup property (for any subset $T$ of $X$, there exists $t_{0} \in T$ such that $\left.A\left(t_{0}\right)=\sup _{t \in T} A(t)\right)$ then $f(A)$ is also a fuzzy filter.

Proof. (1a) Let $x, y \in X, f^{-1}(B)(x \Lambda y)=B(f(x \Lambda y))=B(f(x) \Lambda f(y))$. Since $B$ is a fuzzy filter, we have

$$
B(f(x) \Lambda f(y)) \geq \min (B(f(x)), B(f(y)))=\min \left(f^{-1}(B)(x), f^{-1}(B)(y)\right)
$$

such that $f^{-1}(B)(x \Lambda y) \geq \min \left(f^{-1}(B)(x), f^{-1}(B)(y)\right)$. Similarly, we can prove that

$$
f^{-1}(B)(y \Lambda x) \geq \min \left(f^{-1}(B)(x), f^{-1}(B)(y)\right) .
$$

(b) Let $x, y \in X$ such that $x \leq y$. Then $f(x) \leq f(y) f^{-1}(B)(y)=B(f(y))$. Since $B$ is a fuzzy filter, we have $B(f(y)) \geq B(f(x))=f^{-1}(B)(x)$ such that $f^{-1}(B)(y) \geq$ $f^{-1}(B)(x)$.

(2a) Let $y, y^{\prime} \in Y$ and $x \in f^{-1}(y), x^{\prime} \in f^{-1}\left(y^{\prime}\right)$ such that

$$
\begin{gathered}
A(x)=\sup _{t \in f^{-1}(y)} A(t), \quad A\left(x^{\prime}\right)=\sup _{t \in f^{-1}\left(y^{\prime}\right)} A(t), \\
A\left(x \Lambda x^{\prime}\right)=\sup _{t \in f^{-1}\left(y \Lambda y^{\prime}\right)} A(t), \quad f(A)\left(y \Lambda y^{\prime}\right)=\sup _{t \in f^{-1}\left(y \Lambda y^{\prime}\right)}=A\left(x \Lambda x^{\prime}\right) .
\end{gathered}
$$

Since $A$ is a fuzzy filter,

$$
\begin{aligned}
A\left(x \Lambda x^{\prime}\right) & \geq \min \left(A(x), A\left(x^{\prime}\right)\right) \\
& =\min \left(\sup _{t \in f^{-1}(y)} A(t), \sup _{t \in f^{-1}\left(y^{\prime}\right)} A(t)\right) \\
& =\min \left(f(A)(y), f(A)\left(y^{\prime}\right)\right)
\end{aligned}
$$


such that

$$
f(A)\left(y \Lambda y^{\prime}\right) \geq \min \left(f(A)(y), f(A)\left(y^{\prime}\right)\right) .
$$

Similarly, we can prove that $f(A)\left(y^{\prime} \Lambda y\right) \geq \min \left(f(A)(y), f(A)\left(y^{\prime}\right)\right)$.

(b) Let $y \leq y^{\prime}$ and $x \in f^{-1}(y), x^{\prime} \in f^{-1}\left(y^{\prime}\right)$ such that $A(x)=\sup _{t \in f^{-1}(y)} A(t)$, $A\left(x^{\prime}\right)=\sup _{t \in f^{-1}\left(y^{\prime}\right)} A(t)$, and $x \leq x^{\prime} f(A)\left(y^{\prime}\right)=\sup _{t \in f^{-1}\left(y^{\prime}\right)} A(t)=A\left(x^{\prime}\right)$.

Since $A$ is a fuzzy filter, $A\left(x^{\prime}\right) \geq A(x)=\sup _{t \in f^{-1}(y)} A(t)=f(A)(y)$ such that, $f(A)\left(y^{\prime}\right) \geq f(A)(y)$.

3. Weak filter. In this section, we introduce and discuss the notion of weak filters in a BCI-algebra $X$.

DeFinItion 3.1. Let $A$ be a fuzzy set of $X$. $\tilde{A}$ is a weak filter if for any $x_{\lambda}, y_{\mu} \in \tilde{X}$,

(a) $x_{\lambda} \in \tilde{A}$ and $y_{\mu} \in \tilde{A}$ imply $x_{\lambda} \Lambda y_{\mu} \in \tilde{A}$ and $y_{\mu} \Lambda x_{\lambda} \in \tilde{A}$.

(b) $x_{\lambda} \in \tilde{A}$ and $x_{\lambda} * y_{\mu}=0_{\min (\lambda, \mu)}$ imply $y_{\min (\lambda, \mu)} \in \tilde{A}$.

We characterized fuzzy filters in terms of weak filters as follows.

THEOREM 3.2. A fuzzy subset A of a BCI-algebra $X$ is a fuzzy filter if and only if $\tilde{A}$ is a weak filter.

Proof. Suppose that $\tilde{A}$ is a weak filter, we want to show that $A$ is a fuzzy filter.

(a) Let $x, y \in X$ and $t=\min (A(x), A(y))$. Then $x_{t}, y_{t} \in \tilde{A}$ and because $\tilde{A}$ is a weak filter, we have $x_{t} \Lambda y_{t} \in \tilde{A}$ and $y_{t} \Lambda x_{t} \in \tilde{A}$ such that $A(x \Lambda y) \geq t=\min (A(x), A(y))$ and $A(y \Lambda x) \geq t=\min (A(x), A(y))$.

(b) Let $x, y \in x$ and $x \leq y$, we must show that $A(y) \geq A(x)$.

Let $t=A(x)$. Then $x_{t} \in \tilde{A}$ and because $x \leq y$, we have $x_{t} * y_{t}=0_{t}$.

Using the fact that $\tilde{A}$ is a weak filter, we obtain $y_{t} \in \tilde{A}$ such that $A(y) \geq t=A(x)$. Conversely, assume that $A$ is a fuzzy filter of $X$, we must prove that $\tilde{A}$ is a weak filter.

(a) Let $x_{\lambda}, y_{\mu} \in \tilde{A}$. Then $A(x) \geq \lambda$ and $A(y) \geq \mu$. Since $A$ is a fuzzy filter, $A(x \Lambda y) \geq$ $\min (A(x), A(y)) \geq \min (\lambda, \mu)$ and $A(x \Lambda y) \geq \min (A(x), A(y)) \geq \min (\lambda, \mu)$ such that $x_{\lambda} \Lambda y_{\mu} \in \tilde{A}$ and $y_{\mu} \Lambda x_{\lambda} \in \tilde{A}$.

(b) Let $x_{\lambda} \in \tilde{A}$ and $x_{\lambda} * y_{\mu}=0_{\min (\lambda, \mu)}$. Then $A(x) \geq \lambda$ and $x \leq y$. Because $A$ is a fuzzy filter $A(y) \geq A(x) \geq \lambda \geq \min (\lambda, \mu)$ such that $y_{\min (\lambda, \mu)} \in \tilde{A}$.

The following theorem characterizes weak fuzzy filters (namely, fuzzy filter Theorem 3.2).

THEOREM 3.3. Let A be a closed fuzzy ideal (namely, $\tilde{A}$ is a closed weak ideal Theorem 1.6). Then $\tilde{A}$ is a weak filter if and only if $\tilde{A}$ satisfies the following equivalent conditions:

(i) $x_{\lambda} \in \tilde{A}$ and $y_{\min (\lambda, \mu)} \notin \tilde{A}$ imply that $x_{\lambda} * y_{\mu} \tilde{A}$.

(ii) $x_{\lambda} \in \tilde{A}$ and $x_{\lambda} * y_{\mu} \in \tilde{A}$ imply that $y_{\min (\lambda, \mu)} \in \tilde{A}$.

Proof. It is easy to see that (i) and (ii) are equivalent.

Suppose that $\tilde{A}$ is a weak filter, we want to show that $\tilde{A}$ satisfies (i).

Let $x_{\lambda} \in \tilde{A}$ and $y_{\min (\lambda, \mu)} \notin \tilde{A}$. Using $\left(6^{\prime}\right)$ and $\left(4^{\prime}\right)$, we obtain $0_{\lambda} *\left(x_{\lambda} * y_{\mu}\right)=\left(0_{\lambda} * x_{\lambda}\right) *$ $\left(0_{\lambda} * y_{\mu}\right)=\left[0_{\lambda} *\left(0_{\lambda} * y_{\mu}\right)\right] * x_{\lambda}$. 
If $x_{\lambda} * y_{\mu} \in \tilde{A}$, then $0_{\lambda} *\left(x_{\lambda} * y_{\mu}\right) \in \tilde{A}$ because $\tilde{A}$ is a closed weak ideal. So $\left[0_{\lambda} *\left(0_{\lambda} * y_{\mu}\right)\right] * x_{\lambda} \in \tilde{A}$. Since $x_{\lambda} \in \tilde{A}$ and $\tilde{A}$ is a weak ideal, we obtain $0_{\lambda} *\left(0_{\lambda} * y_{\mu}\right) \in$ $\tilde{A}$. But from $\left(\mathrm{BCI}-3^{\prime}\right)$ and $\left(3^{\prime}\right)\left[0_{\lambda} *\left(0_{\lambda} * y_{\mu}\right)\right] * y_{\mu}=0_{\min (\lambda, \mu)}$ and since $\tilde{A}$ is a weak filter, we obtain $y_{\min (\lambda, \mu)} \in \tilde{A}$ which is a contradiction. Thus $\tilde{A}$ satisfies (i).

Conversely, suppose that $\tilde{A}$ satisfies (ii), we must show that $\tilde{A}$ is a weak filter.

(a) Let $x_{\lambda} \in \tilde{A}$ and $y_{\mu} \in \tilde{A}$. From $\left(7^{\prime}\right)\left(x_{\lambda} \Lambda y_{\mu}\right) * y_{\mu}=0_{\min (\lambda, \mu)}$ and $\left(y_{\mu} \Lambda x_{\lambda}\right) * x_{\lambda}=$ $0_{\min (\lambda, \mu)}$. Because $\tilde{A}$ is a weak ideal, $x_{\lambda} \Lambda y_{\mu} \in \tilde{A}$ and $y_{\mu} \Lambda x_{\lambda} \in \tilde{A}$.

(b) Let $x_{\lambda} \in \tilde{A}$ and $x_{\lambda} * y_{\mu}=0_{\min (\lambda, \mu)}$. Since $\tilde{A}$ is a weak ideal, $x_{\lambda} * y_{\mu} \in \tilde{A}$. Using (ii), we obtain $y_{\min (\lambda, \mu)} \in \tilde{A}$.

4. Closed fuzzy filters. In this section, we introduce and discuss the notion of closed fuzzy filters and closed weak filters in a BCI-algebra.

Definition 4.1 (see [1]). A filter $F$ of $X$ is said to be closed if $0 * x \in F$ when $x \in F$.

Definition 4.2. A fuzzy filter $A$ of $X$ is said to be closed if $A(0 * x) \geq A(x)$ for any $x \in X$.

Definition 4.3. A weak filter $\tilde{A}$ is said to be closed if $0_{\lambda} * x_{\lambda} \in \tilde{A}$ for any $x_{\lambda} \in \tilde{A}$.

Now we discuss the relation between closed filters, closed fuzzy filters and closed weak filters.

THEOREM 4.4. Suppose that $A$ is a fuzzy filter (namely, A is a weak filter; and for any $t \in(0,1]$ the $t$-level subset $A^{t}=\{x \in X / A(x) \geq t\}$ is a filter when $A^{t} \neq \varnothing$, Theorems 2.5 and 3.2). Then the following conditions are equivalent:

(1) $A$ is a closed fuzzy filter.

(2) $\tilde{A}$ is a closed weak filter.

(3) For any $t \in(0,1]$ the $t$-level subset $A^{t}=\{x \in X / A(x) \geq t\}$ is a closed filter when $A^{t} \neq \varnothing$.

Proof. (1) $\Rightarrow(2)$. Let $x_{\lambda} \in \tilde{A}$. Then $A(x) \geq \lambda$. Since $A$ is a closed fuzzy filter, $A(0 * x) \geq$ $A(x) \geq \lambda$ such that $(0 * x)_{\lambda}=0_{\lambda} * x_{\lambda} \in \tilde{A}$.

(2) $\Rightarrow$ (3). Let $x \in A^{t}$. Then $A(x) \geq t$ such that $x_{t} \in \tilde{A}$. Since $\tilde{A}$ is a closed weak filter, $0_{t} * x_{t}=(0 * x)_{t} \in \tilde{A}$. So $A(0 * x) \geq t$ such that $0 * x A^{t}$.

(3) $\Rightarrow(1)$. Let $x \in X$ and $t=A(x)$. Then $x \in A^{t}$ and because $A^{t}$ is a closed filter, we have $0 * x \in A^{t}$ such that $A(0 * x) \geq t=A(x)$.

THEOREM 4.5. Let $A$ be a closed fuzzy filter (namely, $\tilde{A}$ is a closed weak filter Theorem 4.4). Then the following conditions are equivalent.

(i) $A(x * y)=A(y * x)$ for any $x, y \in X$.

(ii) $x_{\lambda} * y_{\mu} \in \tilde{A}$ imply $y_{\mu} * x_{\lambda} \in \tilde{A}$.

Proof. Assume that $A$ is a closed fuzzy filter, we want to show that (ii) holds.

Let $x_{\lambda} * y_{\mu} \in \tilde{A}$. Since $\tilde{A}$ is a closed weak filter, $0_{\lambda} *\left(x_{\lambda} * y_{\mu}\right) \in \tilde{A}$. But $0_{\lambda} *\left(x_{\lambda} * y_{\mu}\right)=$ $[0 *(x * y)]_{\min (\lambda, \mu)}=[(y * y) *(x * y)]_{\min (\lambda, \mu)}=\left(y_{\mu} * y_{\mu}\right) *\left(x_{\lambda} * y_{\mu}\right) \in \tilde{A}$ and using $\left(3^{\prime}\right)$ and $\left(\mathrm{BCI}-1^{\prime}\right)$, we have $\left[\left(y_{\mu} * y_{\mu}\right) *\left(x_{\lambda} * y_{\mu}\right)\right] *\left(y_{\mu} * x_{\lambda}\right)=\left[\left(y_{\mu} * y_{\mu}\right) *\left(y_{\mu} * x_{\lambda}\right)\right] *$ $\left(x_{\lambda} * y_{\mu}\right)=0_{\min (\lambda, \mu)}$. Since $\tilde{A}$ is a weak filter, we obtain $(y * x)_{\min (\lambda, \mu)}=y_{\mu} * x_{\lambda} \in \tilde{A}$. 
Now, we prove that (i) and (ii) are equivalent.

(1) $\Rightarrow(2)$. Let $x_{\lambda}, y_{\mu} \in \tilde{X}$ such that $x_{\lambda} * y_{\mu} \in \tilde{A} . x_{\lambda} * y_{\mu} \in \tilde{A}$ implies $A(x * y) \geq$ $\min (\lambda, \mu)$. But $A(y * x)=A(x * y) \geq \min (\lambda, \mu)$ such that $(y * x)_{\min (\lambda, \mu)}=y_{\mu} * x_{\lambda} \in \tilde{A}$.

(2) $\Rightarrow(1)$. Let $x, y \in X$ and $t=A(x * y)$. Then $(x * y)_{t}=x_{t} * y_{t} \in \tilde{A}$. Using the hypothesis, we obtain $y_{t} * x_{t}=(y * x)_{t} \in \tilde{A}$ such that $A(y * x) \geq t=A(x * y)$.

Similarly, we can prove that $A(x * y) \geq A(y * x)$. Finally, we obtain $A(x * y)=$ $A(y * x)$.

THEOREM 4.6. Theorems 2.7 and 2.9 are valid if "fuzzy filter" is replaced by "closed fuzzy filter."

The proof is the same as Theorems 2.7 and 2.9. So it is omitted.

ACKNowledgement. This work has been supported by the special fund of China for Ph.D. instructors.

\section{REFERENCES}

[1] C. S. Hoo, Filters and ideals in BCI-algebras, Math. Japon. 36 (1991), no. 5, 987-997.

[2] _ Fuzzy ideals of BCI and MV-algebras, Fuzzy Sets and Systems 62 (1994), no. 1, 111-114.

[3] C. S. Hoo and S. Sessa, Fuzzy maximal ideals of BCI and MV-algebras, Inform. Sci. 80 (1994), no. 3-4, 299-309.

[4] W. Huang and F. Liu, On the adjoint semigroups of p-separable BCI-algebras, Semigroup Forum 58 (1999), no. 3, 317-322.

[5] W. P. Huang, On the p-semisimple part in BCI-algebras, Math. Japon. 37 (1992), no. 1, 159-161.

[6] _ On BCI-algebras and semigroups, Math. Japon. 42 (1995), no. 1, 59-64.

[7] Y. B. Jun, Closed fuzzy ideals in BCI-algebras, Math. Japon. 38 (1993), no. 1, 199-202.

[8] C. Lele and C. Wu, Fuzzy weakly prime ideals in BCK-algebras, in preparation.

[9] Characterization of Fuzzy Ideal in BCK-Algebras Using the Notion of Fuzzy Point, Intelligent Techniques and Soft Computing in Nuclear Science and Engineering, World Scientific, Singapore, 2000.

[10] _ Some properties of fuzzy ideals in BCK-algebras, Fuzzy Systems Math. 15 (2001), no. 1, 10-16.

[11]__ Transfer theorems for fuzzy semi-groups, J. Harbin Inst. Tech. 7 (2001), no. 1, $19-23$.

[12] C. Wu and Z. K. Qi, Transfer theorems for fuzzy groups, Fuzzy Math. 2 (1982), no. 1, $51-55$.

[13] C. C. Xi, On a class of BCI-algebras, Math. Japon. 35 (1990), no. 1, 13-17.

C. LeLE: DePARTMENT OF MATHEMATICS, HARBIN INSTITUTE OF TECHNOLOgY, HARbin 150001, CHINA

E-mail address: 1e1e_cle1e@yahoo.com

CONGXin Wu AND T. MAMAdou: Department OF Mathematics, HaRbin INSTITUTE OF TECHNOLOGY, HARBIN 150001, CHINA 


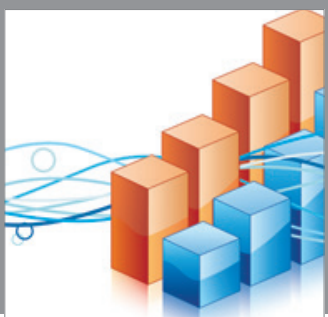

Advances in

Operations Research

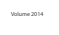

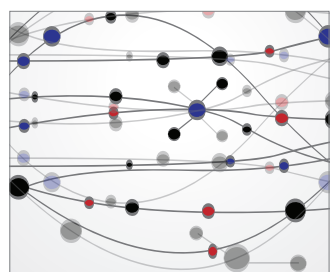

\section{The Scientific} World Journal
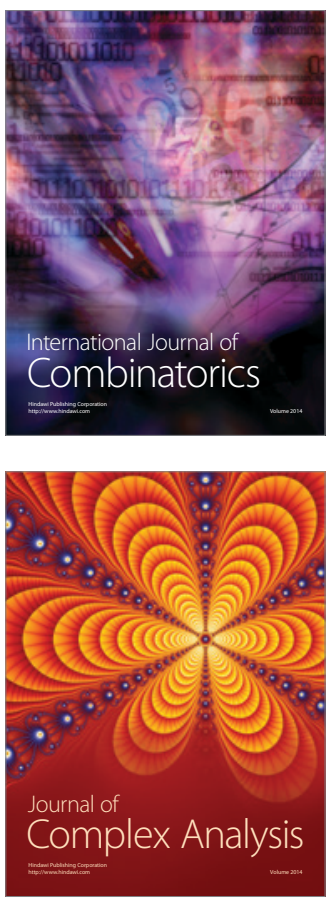

International Journal of

Mathematics and

Mathematical

Sciences
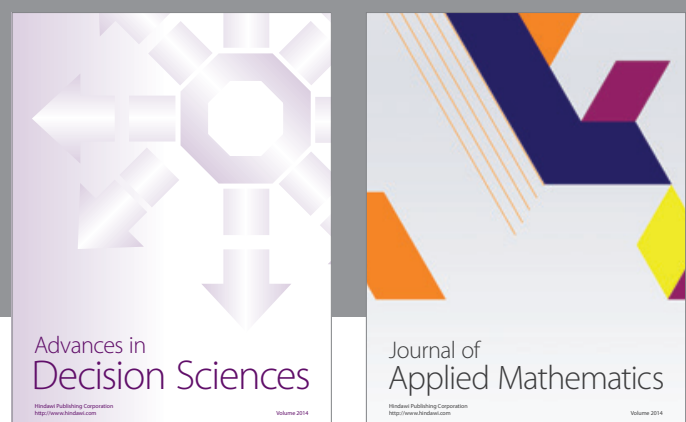

Journal of

Applied Mathematics
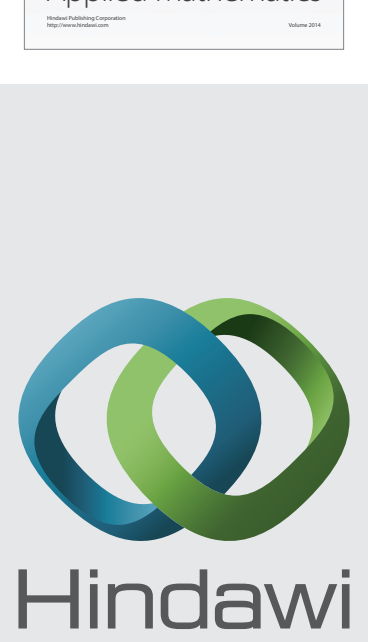

Submit your manuscripts at http://www.hindawi.com
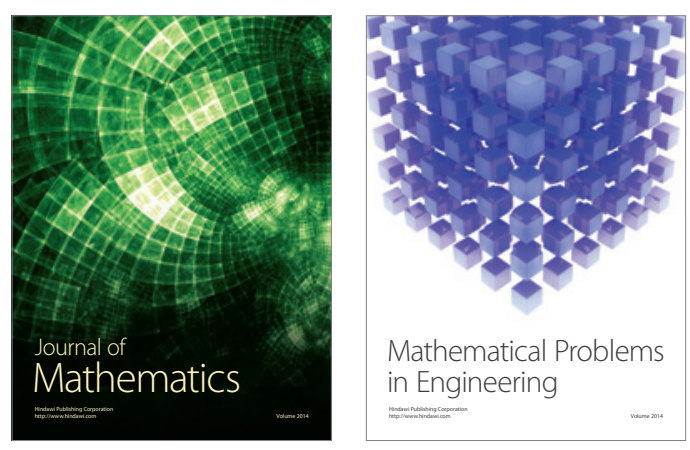

Mathematical Problems in Engineering
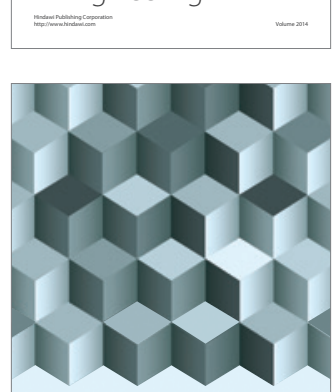

Journal of

Function Spaces
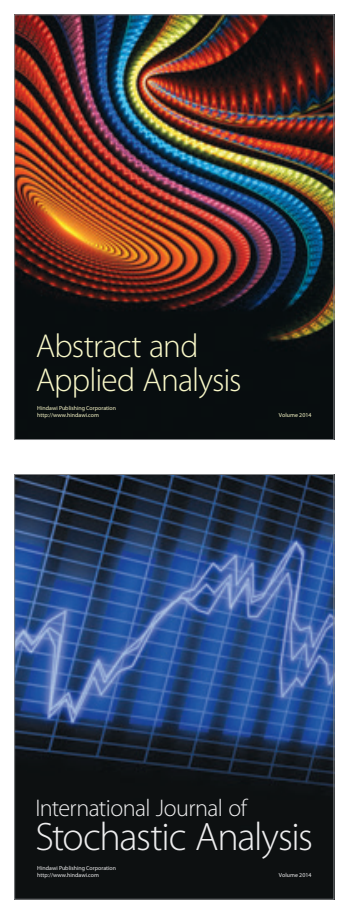

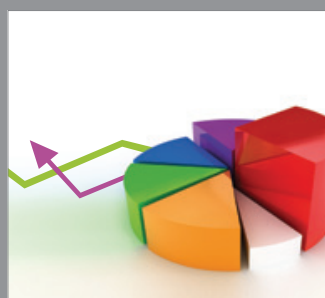

ournal of

Probability and Statistics

Promensencen
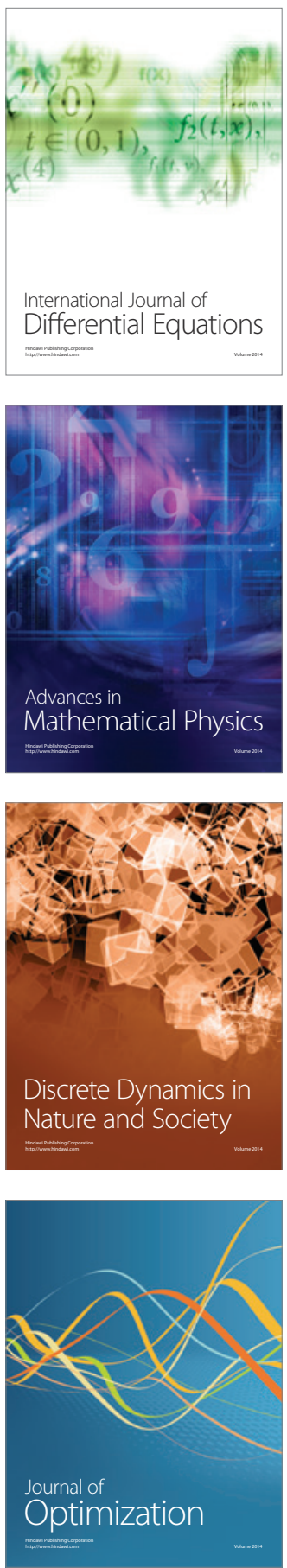